\title{
DNA methylation regulates mouse cardiac myofibril gene expression during heart development
}

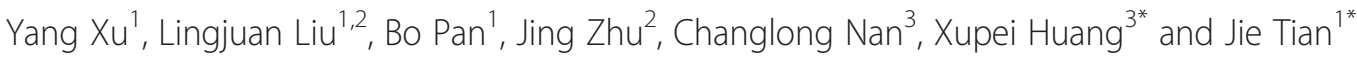

\begin{abstract}
Background: It is well known that epigenetic modifications play an important role in controlling the regulation of gene expression during the development. Our previous studies have demonstrated that the expression of fetal troponin I gene (also called slow skeletal troponin I, ssTnl) is predominated in the fetal stage, reduced after birth and disappeared in the adulthood. The mechanism underlying the developmentally related ssTnl gene regulation is not clear. In this study, we have explored the epigenetic role of DNA methylation in the regulation of ssTnl expression in the heart during the development.
\end{abstract}

Results: The DNA methylation levels of $\mathrm{CpG}$ island and $\mathrm{CpG}$ dinucleotides region were detected using methylation specific PCR (MSP) and bisulfite sequence PCR (BSP) in 2000 bp upstream and 100 bp upstream of ssTnl gene promoter. Real time RT-PCR and Western blot were used to detect ssTnl mRNA and protein expression levels. We found that DNA methylation levels of the CpG dinucleotides region in ssTnl gene promoter were increased with the development, corresponding to a decreased expression of ssTnl gene in mouse heart. However the DNA methylation levels of $\mathrm{CpG}$ islands in this gene were not changed during the development. Application of a methylation inhibitor, 5-Azacytidine, in cultured myocardial cells partially prevented the decline of ssTnl expression.

Conclusion: Our results indicate that DNA methylation, as an epigenetic intervention, plays a role in the regulation of the fetal Tnl gene expression in the heat during the development.

Keywords: Troponin I, DNA methylation, 5-Azacytidine, Epigenetic regulation

\section{Background}

Sarcomere protein, troponin, is located on the thin filament of myocardial cells and plays an essential role in regulating $\mathrm{Ca}^{2+}$-activated tension of striated muscles. Troponin component contains three isoforms: troponin $\mathrm{T}(\mathrm{TnT})$, binding to tropomyosion forming troponintropomyosion complex, troponin $\mathrm{C}$ ( $\mathrm{TnC}$ ), binding to $\mathrm{Ca}^{2+}$ to produce a conformational change in $\mathrm{TnT}$, and troponin I (TnI), an inhibitory subunit binding to actintropomyosion and regulating muscles contraction [1]. There are at least two developmentally regulated TnI

\footnotetext{
* Correspondence: xhuang@fau.edu; jietian@cqmu.edu.cn

${ }^{3}$ Department of Biomedical Science, Charlie E. Schmidt College of Medicine, Florida Atlantic University, 777 Glades Road, Boca Raton, FL 33431, USA

'Department of Cardiology, Heart Centre, Children's Hospital of Chongqing Medical University, 136 Zhongshan 2nd Road, Yu Zhong District, Chongqing 400014, P.R. of China

Full list of author information is available at the end of the article
}

isoforms in the heart: the slow skeletal TnI (ssTnI) that is expressed in the fetal heart and the cardiac TnI (cTnI) that is predominately expressed in adult hearts $[1-3]$. TnI isoform switching is common in animals and human, and it is a good model to investigate the regulation of cardiac proteins in the development [4].

It is clinically important to clarify the regulation of $\mathrm{TnI}$ expression, because many cardiomyopathy and heart diseases are associated with abnormal TnI protein expression leading to diastolic dysfunction and heart failure [5-7]. In our previous studies, we have demonstrated that ssTnI expression in heart is partially regulated by thyroid hormone during the heart development $[8,9]$. And we have also cloned mouse ssTnI gene with upstream promoters and revealed several regions and domains on the promoters critically to ssTnI gene expression, such as TnI slow upstream regulatory elements (SURE), Yin Yang 1 factor (YY1), proximal $300 \mathrm{bp}$ upstream region and the first 
intron of ssTnI gene [10-13]. However, the mechanisms of ssTnI down-regulation and finally shut down in the heart after birth is still not clear.

DNA methylation is one of epigenetic modifications and many studies have showed that DNA methylation plays an important role in gene expression, genomic imprinting, X-chromosome inactivation and chromatin structure changes [14-16]. DNA methylation occurs on position 5 of cytosine by the covalent modification of a methyl group, creating 5-methylcytosine, which is preferentially found in $\mathrm{CpG}$ dinucleotides [17]. The "CpG" is shorthand for "-C-phosphate-G-", which is cytosine and guanine separated by only one phosphate. CpG island is a region with high frequency of CpG sites (GC percentage is greater than $50 \%$ in a region over $200 \mathrm{bp}$ ). In addition, some CpG sites that are in a short distance from $\mathrm{CpG}$ island are called $\mathrm{CpG}$ island sites (or CpG shore), which also play an important role in regulating gene expression [18]. The methylation of CpG dinucleotides in promoter of genes always leads to a transcriptional inactivation. Some studies have shown that different levels or patterns of cytosine methylation are found in various tissues or in different functional regions of the same tissue [19-21]. Our previous study has shown that epigenetic modification may regulate ssTnI expression during heart development, especially through histone acetylation and histone methylation [22]. However, the role of DNA methylation in the regulation of ssTnI gene expression is still unknown. In the present study, we have measured the levels of DNA methylation in critical domains of ssTnI genes to determine the role of epigenetic regulation on this gene expression. Furthermore, a DNA methyltransferase inhibitor, 5-azacytidine has been applied to myocardial cells to confirm the DNA methylation mediated ssTnI gene expression in the heart. Our results indicate that DNA methylation plays an important role in the regulation of ssTnI expression in the heart during the development.

\section{Methods}

\section{Experimental animals}

All animal procedures were approved by the Animal Care and Use Committee at the Chongqing Medical University. Adult wild type KM mice (body weight of 18-22 g) were purchased from Chongqing Medical University Animal Center. Upon arrival, breeder mice were separately housed and acclimated for at least one week before mating began. The mice were maintained on a reverse 12 h light-dark cycle (light 19:00-07:00) and provided with laboratory chow and water ad libitum. Two females were placed with one male for two hours between 08:00 and 10:00. When a vaginal plug was detected after mating period, which was designated as embryonic 0.5 day (E0.5). E14.5, E17.5 pregnant mice, postnatal day 1 , day 7 , day 14 and adult mice were sacrificed using $\mathrm{CO}_{2}$ gas. Embryonic and postnatal heart tissue were collected and frozen at $-80{ }^{\circ} \mathrm{C}$ until use.

\section{Culture of mouse myocardial cells}

Primary cultures of myocardial cells were performed as described previously [23]. Cardiac myocytes were collected from the mice of postnatal day 14 mice. The cells were randomly divided into two groups, the untreated control group (treated with nothing), 5-azacytidine group (5 $\mu$ M 5-azacytidine). 5-azacytidine (Sigma, Santa Clara, California, USA) was dissolved in tissue culture medium to make a stock solution and stored in $-20{ }^{\circ} \mathrm{C}$. After treated with 5-azacytidine for $24 \mathrm{~h}$, cells were collected and stock at $-80{ }^{\circ} \mathrm{C}$ for further analyses.

\section{Real-Time RT-PCR}

The real-time PCR was carried out as described previously $[10,11]$. The data were analyzed using $2^{-\left[\Delta \mathrm{Ct} \text { (target) }-{ }^{\Delta} \mathrm{Ct}\right.}$ (input)] method [24] following the manufacture's instruction. The primers for PCR amplification: ssTnI forwarding primer: 5 ' -CTCCACGAGGACTAAACTAGGC-3', reversing primer: 5'-CTTGGATTTCCTCTCAACTTCC-3'. $\beta$ actin forwarding primer: 5'-CACACCCGCCACCAG TTCG-3', reversing primer : 5'-GTCCTTCTGACCCAT TCCCACC-3'.

\section{Western blot}

Western blotting assays for ssTnI were performed as previously describe [8]. An anti-TnI monoclonal antibody (TnI-1) that recognized mouse ssTnI was used at a dilution of 1:10,000. The immune-reactive protein bands were visualized with Chemiluminescent Luminol Reagent (Merck Millipore, USA). After scanning, protein bands were analyzed with Quantity One Version 4.4 software (Bio-Rad, CA, USA).

\section{Methylation specific PCR (MSP)}

Genomic DNA was isolated from ventricular tissue and myocytes cells using a TIANamp Genomic DNA Kit (Tiangen, Beijing, China). DNA methylation of ssTnI promoter $\mathrm{CPG}$ islands and $\mathrm{CpG}$ dinucleotides region were detected by methylation specific PCR (MSP) using $2 \times$ Power Taq PCR MasterMix (Bioteck, Beijing, China) in a final volume of $25 \mu \mathrm{l}$. Bisulfite treatment of unmethylated DNA converted cytosines to uracils at CpG dinucleotides. However, methylated cytosines were not converted. Specific primers were designed to amplify the target regions of interest with unmethylated $\mathrm{CpG}$ dinucleotides by detecting uracils and methylated CpG dinucleotides by detecting cytosines. Primer were designed using MethPrimer software (http://www.urogene.org/cgi-bin/methprimer/methprimer.cgi). M pair was indicated methylation of $\mathrm{CpG}$ sites within the primer sequences, $\mathrm{U}$ pair indicated 
no methylation, and both pairs indicated a partial methylation: ssTnI $\mathrm{CpG}$ island forwarding $\mathrm{M}$ primer: 5'-TTGGGGTAGTAGGGTAGAGATATTC-3', reversing M primer: 5' -TTCTCTTATTCTAAATTCCAACGTC-3'. Forwarding U primer: 5' -TTGGGGTAGTAGGGTAGAG ATATTT-3', reversing U primer: 5'-TTCTCTTATTCT AAATTCCAACATC-3'. ssTnI CpG dinucleotides region forwarding $M$ primer: 5'-ACGGTAGTATATATTTGTT TTGCGA-3', reversing M primer: 5'-ACTATAAAAACC GTAACCTCCGAC-3'. Forwading U primer: 5'-ATGGT AGTATATATTTGTTTTGTGA-3', reversing U primer: 5' -AACTATAAAAACCATAACCTCCAAC-3'.

\section{Bisulfite Sequencing PCR (BSP)}

To verify the methylation level of these heart tissues and myocytes cells, bisulfite sequencing PCR (BSP) was used. DNA was first modified by treatment with sodium bisulfite to convert all 'C's to uracil residues except $5 \mathrm{mCs}$. Then bisulfite-modified DNA were amplified by PCR, which performed in a RT-PCR instrument (MJ Mini Personal Thermal Cycler, BIO-RAD) using $2 \times$ Power Taq PCR MasterMix (Bioteck, Beijing, China) under the Touch-down program: $95{ }^{\circ} \mathrm{C}$ for 3 mins, followed by 9 cycles of $94{ }^{\circ} \mathrm{C}$ for $30 \mathrm{~s}, 60^{\circ} \mathrm{C}$ for $30 \mathrm{~s}$ (decrease $1{ }^{\circ} \mathrm{C}$ per cycle), $72{ }^{\circ} \mathrm{C}$ for $1 \mathrm{~min}$, then followed by 40 cycles of $94{ }^{\circ} \mathrm{C}$ for $30 \mathrm{~s}, 50{ }^{\circ} \mathrm{C}$ for $30 \mathrm{~s}, 72{ }^{\circ} \mathrm{C}$ for $1 \mathrm{~min}$, and final extension $72{ }^{\circ} \mathrm{C}$ for 10 mins. The BSP primers were designed using MethPrimer: ssTnI CpG island forwarding primer: 5'-TGGGGTTAGAGTGTAAAGTTAATATTG-3', reversing primer: 5'-TATAACTCCAAACACCCATCTC TCT-3'. ssTnI CpG dinucleotides region forwarding primer: 5' -TTGGTTTTTAAGTTTGTGGTTTATA-3', reversing primer: $5^{\prime}$-CTAAACTAACCTAAACCTCACCAC AA-3'. The resulted PCR product were used to direct sequencing at INVITROGEN (Shanghai, China) to examine bisulfite conversion rate for different time heart tissue [25]. Moreover, the PCR product were recovered by TIANgel Midi Purification Kit (TIANGEN, Beijing, China) after verification in a $2 \%$ agarose gel. Then the purified DNA was ligated into the vector pGM-T by pGM-T Cloning Kit with Competent Cell (TIANGEN, Beijing, China) and transformed into $E$. coli strain TOP10. Sequence determinations were carried out at SANGON (Shanghai, China) [14].

\section{Statistics}

All RT-PCR and Western blot data are showed as mean \pm SD. BSP sequences data are analyzed using software from BiQ Analyzer (http://biq-analyzer.bioinf.mpi-inf.mpg.de/) to calculate the level of DNA methylation (methylation level $=$ methylated $\mathrm{CpG}$ dinucleotides/total $\mathrm{CpG}$ dinucleotides). Statistical analysis was carried out by SPSS 19.0 using ANOVA and Student's $t$-test to determine statistical significance. The criteria for significance were defined as $\mathrm{p}<0.05$.

\section{Results}

The time course of the expression of ssTnI mRNA and protein in mouse heart during embryonic day 14.5 to adult is shown in Fig. 1. The highest expression level of ssTnI mRNA was observed in embryonic day 14.5 and 17.5. The expression of ssTnI was declined gradually after birth. In the adult heart, ssTnI expression was barely detected (Fig. 1a). The time course of ssTnI protein levels (Fig. 1b and c) was similar to that of ssTnI mRNA during the heart development, which is consistent with what we reported previously [8].

The MethPrimer was used to predict $\mathrm{CpG}$ islands and CpG dinucleotides regions in ssTnI gene promoter. Only one CpG island was found in the upstream of ssTnI gene, which is about 2000 bp away from the transcription start site (TSS). However, various CpG dinucleotides were found in different domains of the ssTnI gene promoter. One $\mathrm{CpG}$ dinucleotides region containing 5 CpG sites was found in about $100 \mathrm{bp}$ from TSS on ssTnI gene. MSP assays were performed to determine DNA methylation on ssTnI gene promoter. The DNA methylation of $\mathrm{CpG}$ island in ssTnI was shown in Fig. 2a, indicating that all of $\mathrm{CpG}$ islands were fully methylated in all of the time points tested during the heart development. However, the methylation of $\mathrm{CpG}$ dinucleotides region was different, which showed that the $\mathrm{CpG}$ sites was unmethylated in embryonic day 14.5, 17.5 and newborn, but after birth on postnatal day 7 and 14, methylation occurred on these sites and reached a peak level of methylation in adulthood (Fig. 2b). The gradual methylation of these sites on ssTnI gene shortly after birth is corresponding to a gradual decline of the ssTnI gene expression in the heart during the same time course.

Bisulfite sequence PCR (BSP) assays were carried out to confirm the data obtained from MSP assays. The data from BSP showed that DNA methylation in CpG island of ssTnI gene was not significantly changed during heart development, however, the DNA methylation levels in CpG dinucleotides regions showed that these sites were not methylated during the embryonic stage and even at newborn in the heart and methylation started on these site shortly after the birth, which are consistent with the results from MSP assays.

5-azacytidine, a methylation inhibitor, was applied to 14-day-old myocardial cells to see whether ssTnI expression increases after the inhibition of ssTnI gene methylation. The expression of mRNA in myocardial cells that were prior treated with $5 \mu \mathrm{M} 5$-azacytidine for $24 \mathrm{~h}$ was significantly increased compared to that of the untreated control group (Fig. 3). The MSP data showed that 5azacytidine could reduce DNA methylation in the CpG dinucleotides regions, while the methylation status of $\mathrm{CpG}$ island was not changed compared to the untreated control group (Fig. 4a and c). The BSP data in myocardial cells 


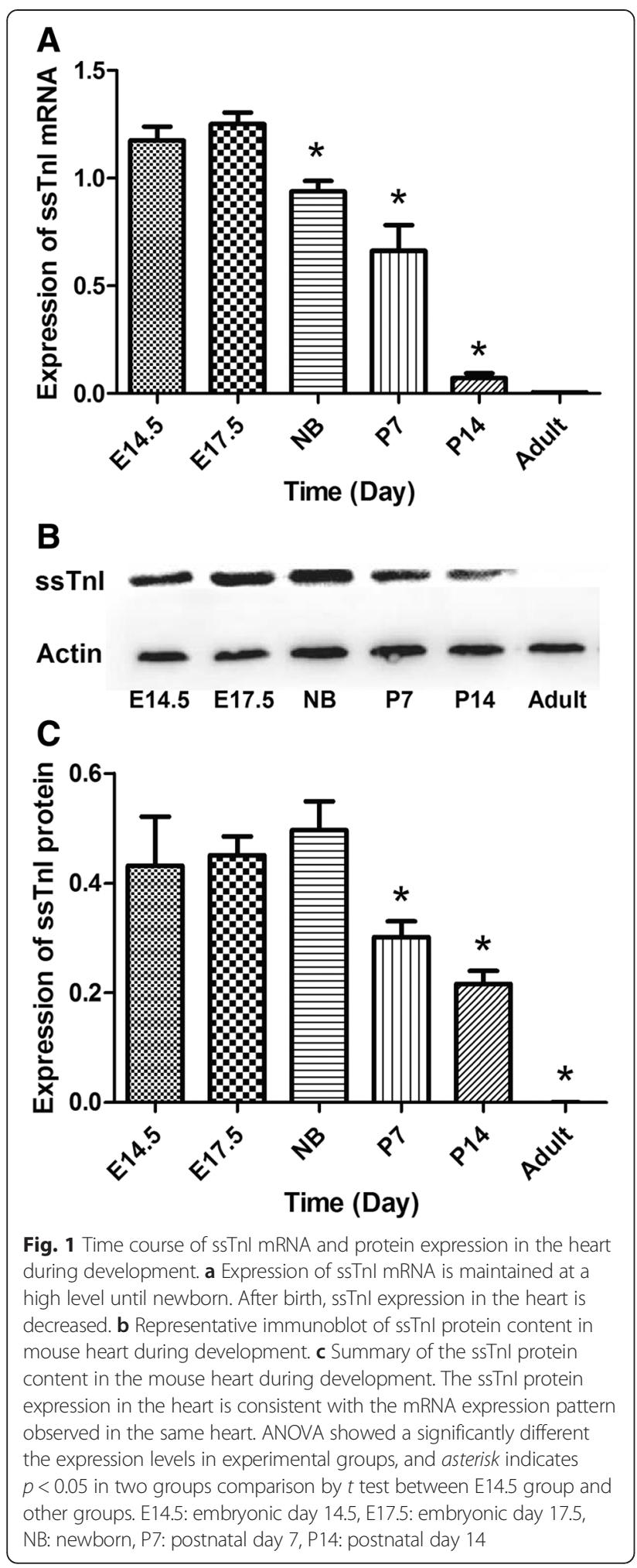

indicated that 5-azacytidine could reduce the methylation level of $\mathrm{CpG}$ dinucleotides region in ssTnI promoter compared to the untreated control group (Fig. $4 \mathrm{~b}$ and d).

\section{Discussion}

Troponin I is not only a structural protein but also an important regulatory protein that controls cardiac muscle contraction and relaxation. The ischemia and oxidative stress may cause cardiac muscle cell damages such as cell death, and then, the troponin protein is released from myocardial cells into blood. Therefore, troponin has become a critical myocardial marker to estimate the cardiac damages [26, 27]. Troponin I isoform switching during heart development was discovered almost three decades ago [28]. But the mechanisms of the fetal TnI (ssTnI) gene inactivation soon after birth are still unclear. The developmental switch from fetal to adult troponin I is found in lower and higher animals, and even in human beings. For this reason, TnI family has become a good model to investigate the regulation of cardiac proteins during heart development [4]. Re-expression of many fetal protein genes normally observed in fetal heart may occur in several pathological conditions such as cardiac hypertrophy and heart failure [29]. However no ssTnI re-expression is observed even in severe pathological conditions or in the heart at the end stage $[9,30]$. We have cloned and characterized mouse ssTnI gene and our previous studies have showed that ssTnI gene was gradually shut down 15 days after birth in mouse hearts. Several important regulatory domains and elements on ssTnI promoters have been identified, such as SURE, a specific domain in upstream part of ssTnI gene, and one internal regulatory element (IRE) in the first intron region [4, 10-13]. We also predicted some transcription factors binding to the $100 \mathrm{bp}$ upstream of ssTnI gene promoter, for example, specificity protein 1 (Sp1), myogenic differentiation (MyoD) and myocyte enhancer factor-2 (MEF-2). In addition, we have also found that the thyroid hormone could partially regulate the ssTnI gene expression in the developing mouse heart [9].

Recently, epigenetics, which is defined as the interaction of DNA methylation, histone modification and expression of noncoding RNAs, has been demonstrated to play an important role in the regulation of gene expression during the development [31-33]. Early studies have showed that methylation and demethylation play a role on regulation of gene expression during development, and there is an inverse correlation between DNA methylation and gene expression [33-35]. In mammalian genome, approximately $70 \%$ of $\mathrm{CpG}$ are methylated [36], on the other hand, unmethylated CpG are grouped in clusters called "CpG island" in gene promoter regulatory regions. Interestingly, some methylation alterations do not occur in $\mathrm{CpG}$ island but in $\mathrm{CpG}$ dinucleotides (called $\mathrm{CpG}$ shores), and methylation on $\mathrm{CpG}$ island shores is strongly related to gene expression [18]. Our 

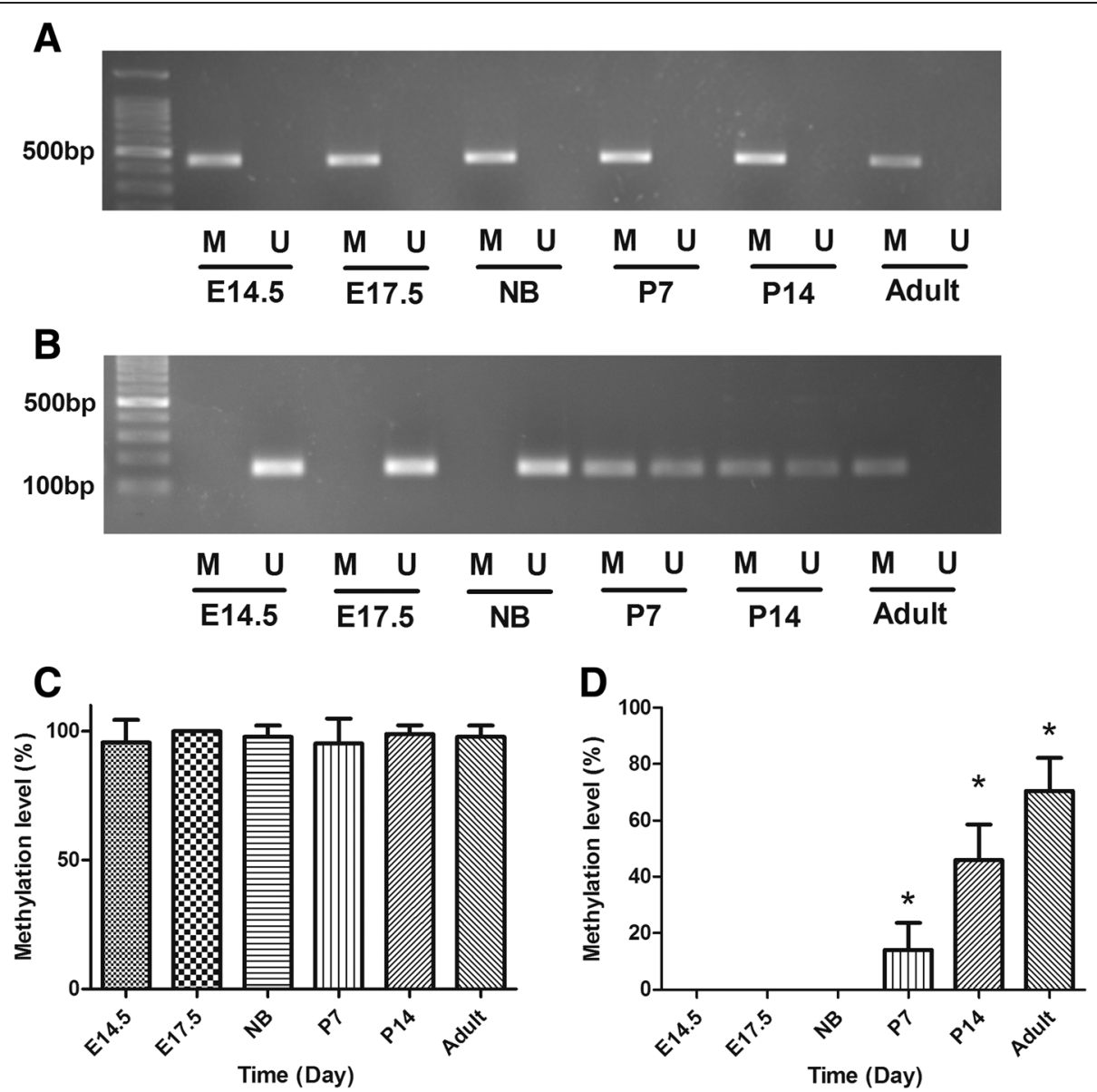

Fig. 2 DNA methylation of CpG islands and CpG dinucleotide regions in ssTnl gene promoter. a DNA methylation of CpG islands in ssTnl promoter. The CpG island is fully methylated in the cardiac tissues at different time points during development. b CpG dinucleotides methylation in -100 bp region away from ssTnl transcription starting site (TSS). In E14.5, E17.5 and NB, the CpG dinucleotides are unmethylated, but in P7, P14, those regions show a partial methylation. In adult, it is fully methylated. $M$, methylated; $U$, unmethylated. c The ssTnl CpG island methylation levels observed in mouse cardiac tissues during development. Methylation on ssTnl CpG islands is stably maintained in a high level during heart development. $\mathbf{d}$ CpG dinucleotides methylation levels observed in ssTnl promoter -100 bp. The bisulfite sequence data show that an altered DNA methylation level of $\mathrm{CpG}$ site in SsTnl -100 bp region during heart development. ANOVA analyses indicate a significantly different in methylation levels among various experimental groups, and ${ }^{*} p<0.05$ indicates a statistical difference in two groups compared using $t$ test

previous study has shown that histone modification, such as acetylation or methylation, may affect ssTnI expression during heart development [22]. However, little is known about the effect of DNA methylation on the regulation of ssTnI gene expression during heart development. In this study, our results have demonstrated that the DNA methylation on CpG dinucleotides regions of ssTnI promoter, located on 100 bp upstream from TSS, is gradually increased during heart development, but the level of DNA methylation on CpG island of ss'TnI promoter does not change, suggesting the DNA methylation on $\mathrm{CpG}$ dinucleotides regions is associated with a down regulation of ssTnI gene expression in the heart. This $\mathrm{CpG}$ dinucleotides region is located in $100 \mathrm{bp}$ upstream of the ssTnI TSS, which has been identified to be able to bind with several critical transcription factors, such as Sp1, MyoD and MEF-2. The enhanced methylation of this domain may inhibit the function of transcription factors and affect gene expression. Whereas the methylation of $\mathrm{CpG}$ island on ssTnI promoters is stable without any significant change at the different time points during the development, indicating that the CpG islands may not be the targets for regulation of ssTnI gene expression during heart development.

5 '-azacytidine is a DNA methyl-transferase inhibitor that can reverse the DNA hypermethylation and reestablish gene expression $[37,38]$. In this study, in order to confirm the methylation regulatory mechanism, 5-azacytidine has been used to treat myocardial 


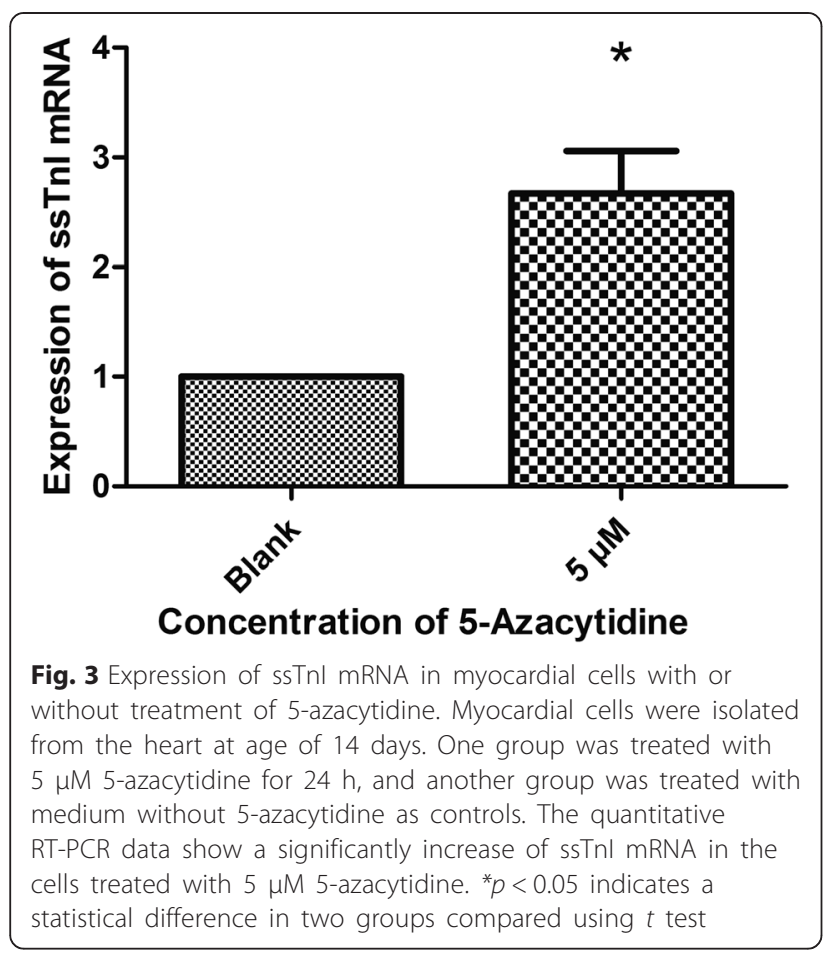

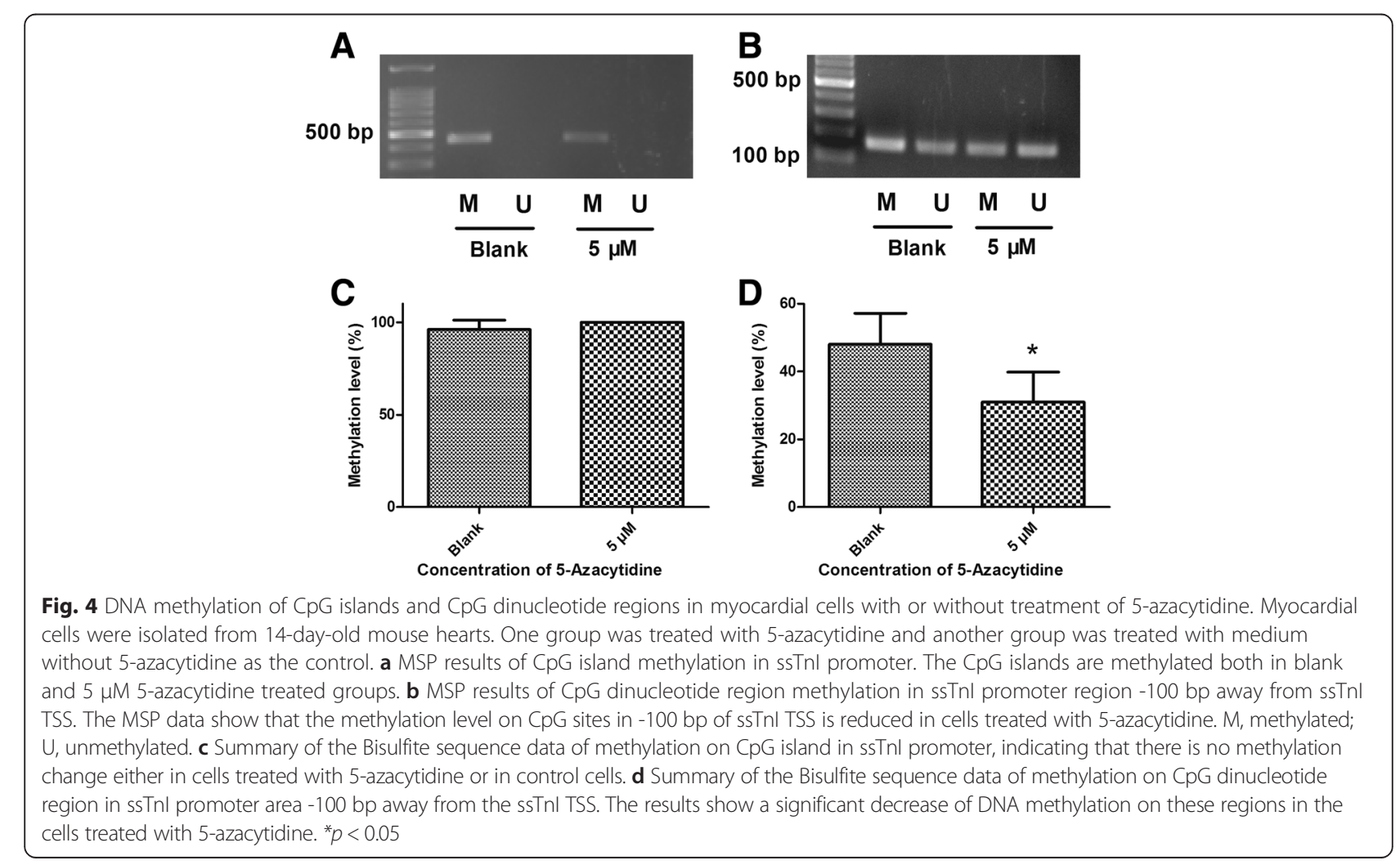

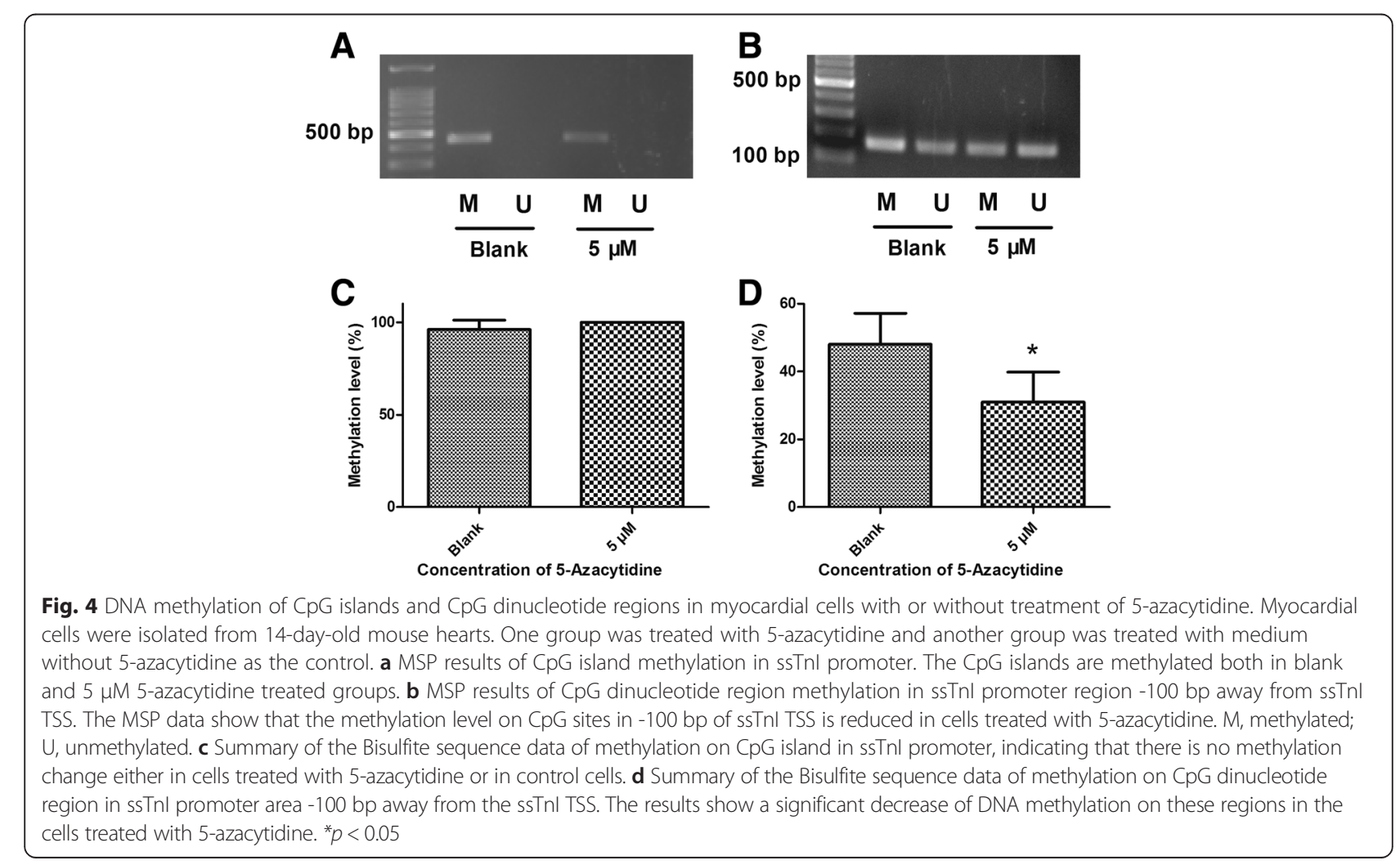

cells to see whether ssTnI expression can be resumed by 5 -azacytidine. Our results indicate that expression of ssTnI mRNA is increased in myocardial cells after treatment with 5 -azacytidine $(5 \mu \mathrm{M})$. The MSP and BSP data have shown that the increased DNA methylation in dinucleotides region located in $-100 \mathrm{bp}$ of ssTnI promoter is significantly reduced in myocardial cells treated with 5azacytidine, while the $\mathrm{CpG}$ island was not influenced. Our study has demonstrated for the first time that methylation on the $\mathrm{CpG}$ dinucleotides region, not the $\mathrm{CpG}$ island, may play an important role in regulation of ssTnI gene expression during heart development.

\section{Conclusion}

Our results indicate that DNA methylation occurs on CpG dinucleotides region, locating at $-100 \mathrm{bp}$ of ssTnI gene promoter, can cause the corresponding changes in ssTnI gene expression. But the methylation of $\mathrm{CpG}$ island on ssTnI gene promoter may not be critical in the regulation of ssTnI gene expression during heart development. In addition, DNA methylation inhibitor 5-azacytidine can increase ssTnI mRNA expression by reducing methylation on $\mathrm{CpG}$ dinucleotides region in ssTnI gene promoter. These data indicate that the epigenetic modification is involved in regulation of myofibril gene expression during heart development in mice. 


\section{Competing interests}

The authors declare that they have no competing interests.

\section{Author's contribution}

YX, LL and BP have performed Western blotting assays and ChIP assays in the study. CN was participated in the primer design and real-time PCR experiments. $\mathrm{YX}, \mathrm{JZ}, \mathrm{XH}$ and JT were participated in the experimental design, data analysis and manuscript preparation. All authors read and approved the final manuscript.

\section{Acknowledgments}

This study was supported by research grants from Natural Science Foundation of China (NSFC Grant Number: 31271218 to XH).

\section{Author details}

'Department of Cardiology, Heart Centre, Children's Hospital of Chongqing Medical University, 136 Zhongshan 2nd Road, Yu Zhong District, Chongqing 400014, P.R. of China. ${ }^{2}$ Ministry of Education Key Laboratory of Child Development and Disorders; Key Laboratory of Pediatrics in Chongqing, CSTC2009CA5002; Chongqing International Science and Technology Cooperation Center for Child Development and Disorders, Chongqing, P.R. of China. ${ }^{3}$ Department of Biomedical Science, Charlie E. Schmidt College of Medicine, Florida Atlantic University, 777 Glades Road, Boca Raton, FL 33431, USA.

\section{Received: 3 August 2015 Accepted: 9 October 2015}

\section{Published online: 17 October 2015}

\section{References}

1. Tobacman LS. Thin filament-mediated regulation of cardiac contraction. Annu Rev Physiol. 1996:58:447-81.

2. Ausoni S, De Nardi C, Moretti P, Gorza L, Schiaffino S. Developmental expression of rat cardiac troponin I mRNA. Development. 1991;112:1041-51.

3. Westfall MV, Rust EM, Metzger JM. Slow skeletal troponin I gene transfer, expression, and myofilament incorporation enhances adult cardiacmyocyte contractile function. Proc Natl Acad Sci U S A. 1997;94(10):5444-9.

4. Zhu L, Lyons GE, Juhasz O, Joya JE, Hardeman EC, Wade R. Developmental regulation of troponin I isoform genes in striated muscles of transgenic mice. Dev Biol. 1995;169:487-503.

5. Gao WD, Atar D, Liu Y, Perez NG, Murphy AM, Marban E. Role of troponin I proteolysis in the pathogenesis of stunned myocardium. Circ Res. 1997:80:393-9.

6. Hunkeler NM, Kullman J, Murphy AM. Troponin I isoform expression in human heart. Circ Res. 1991;69:1409-14.

7. Liu J, Du J, Zhang C, Walker JW, Huang X. Progressive troponin I loss impairs cardiac relaxation and causes heart failure in mice. Am J Physiol Heart Circ Physiol. 2007;293:1273-81.

8. Huang X, Pi Y, Lee KJ, Henkel AS, Gregg RG, Powers PA, et al. Cardiac troponin I gene knockout: a mouse model of myocardial troponin I deficiency. Circ Res. 1999;84:1-8.

9. Huang X, Lee KJ, Riedel B, Zhang C, Lemanski LF, Walker JW. Thyroid hormone regulates slow skeletal troponin I gene inactivation in cardiac troponin I null mouse hearts. J Mol Cell Cardiol. 2000;32:2221-8.

10. Du J, Nan C, Huang JJ, Zhang C, Liu J, Jia P, et al. Functional characterization of mouse fetal $\mathrm{Tnl}$ gene promoters in myocardial cells. J Biomed Sci. 2008;15:605-13.

11. Nan C, Huang X. Transcription factor Yin Yang 1 represses fetal troponin I gene expression in neonatal myocardial cells. Biochem Biophys Res Commun. 2009;378:62-7.

12. Nakayama M, Stauffer JS, Cheng J, Banerjee-Basu S, Wawrousek E, Buonanno A. Common core sequences are found in skeletal muscle slow-, fast-fiber-typespecific regulatory elements. Mol Cell Biol. 1996;16:2408-17.

13. Calvo S, Venepally P, Cheng J, Buonanno A. Fiber-type-specific transcription of the troponin I slow gene is regulated by multiple elements. Mol Cell Biol. 1999;19:515-25.

14. Yang C, Zhang M, Niu W, Yang R, Zhang Y, Qiu Z, et al. Analysis of DNA methylation in various swine tissues. PLoS One. 2011;6(1):e16229.

15. Razin A, Cedar H. DNA methylation and gene expression. Microbiol Rev. 1991;55:451-8
16. Chen B, Kung HF, Bates RR. Effects of methylation of the betagalactosidase genome upon in vitro synthesis of beta-galactosidase. Chem Biol Interact. 1976;14:101-11.

17. Brena RM, Huang TH, Plass C. Toward a human epigenome. Nat Genet. 2006;38(12):1359-60.

18. Irizarry RA, Ladd-Acosta C, Wen B, Wu Z, Montano C, Onyango P. et.al, Genome-wide methylation analysis of human colon cancer reveals similar hypo- and hypermethylation at conserved tissuespecific CpG island shores. Nat Genet. 2009;41(2):178-86.

19. Vanyushin BF. Enzymatic DNA methylation is an epigenetic control for genetic functions of the cell. Biochemistry (Mosc). 2005;70:488-99.

20. Bird A. DNA methylation patterns and epigenetic memory. Genes Dev. 2002;16:6-21.

21. Mandel JL, Chambon P. DNA methylation: organ specific variations in the methylation pattern within and around ovalbumin and other chicken genes. Nucleic Acids Res. 1979;7:2081-103.

22. Zhao W, Liu L, Pan B, Xu Y, Zhu J, Nan C, et al. Epigenetic regulation of cardiac myofibril gene expression during heart development. Cardiovasc Toxicol. 2014;15(3):203-9.

23. Sreejit $P$, Kumar $S$, Verma RS. An improved protocol for primary culture of ardiomyocyte from neonatal mice. In Vitro Cell Dev Biol Anim. 2008;44(3-4):45-50.

24. Schmittgen TD, Livak KJ. Analyzing real-time PCR data by the comparative CT method. Nat Protoc. 2008;3(6):1101-8.

25. Li LC, Dahiya R. MethPrimer: designing primers for methylation PCRs. Bioinformatics. 2002;18(11):1427-37.

26. Chapelle JP. Cardiac troponin I and troponin T: recent players in the field of yocardial markers. Clin Chem Lab Med. 2005:37:11-20.

27. $\mathrm{Wu} \mathrm{AH}$, Christenson $\mathrm{RH}$. Analytical and assay issues for use of cardiac troponin esting for risk stratification in primary care. Clin Biochem. 2013;46:969-78

28. Sasse S, Brand NJ, Kyprianou P, Dhoot GK, Wade R, Arai M, et al. Troponin I gene expression during human cardiac development and in end-stage heart failure. Circ Res. 1993;72:932-8.

29. Boheler KR, Schwartz K. Gene expression in cardiac hypertrophy. Trends Cardiovasc Med. 1992;2:176-82.

30. Cumming DV, Seymour AM, Rix LK, Kellett R, Dhoot GK, Yacoub MH, et al. Troponin I and T protein expression in experimental cardiac hypertrophy. Cardioscience. 1995;6:65-70.

31. Jones PA, Baylin SB. The fundamental role of epigenetic events in cancer Nat Rev Genet. 2002;3:415-28.

32. Shilatifard A. Chromatin modifications by methylation and ubiquitination: implications in the regulation of gene expression. Annu Rev Biochem. 2006:75:243-69.

33. Holliday R, Pugh JE. DNA modification mechanisms and gene activity during development. Science. 1975;187:226-32.

34. Weber M, Hellmann I, Stadler MB, Ramos L, Paabo S, Rebhan M, et al. Distribution, silencing potential and evolutionary impact of promoter DNA methylation in the human genome. Nat Genet. 2007;39:457-66.

35. Riggs AD. X inactivation, differentiation, and DNA methylation. Cytogenet Cell Genet. 1975;14:9-25.

36. Issa JP. CpG island methylator phenotype in cancer. Nat Rev Cancer. 2004;4:988-93.

37. Enver T, Zhang JW, Papayannopoulo T, Stamatoyannopoulos G. DNA methylation: a secondary event in globin gene switching? Genes Dev. 1998;2:698-706.

38. Esteller M. Epigenetics in cancer. N Engl J Med. 2008;358:1148-59. 